\section{Brain, Behavior and Evolution}

\title{
Preface: Evo-Devo on the Brain
}

Although Darwin and other pioneers of evolutionary biology speculated about the relationship between organismal development and evolution, embryology was omitted from the evolutionary synthesis that emerged in the 1940s. This began to change in the 1980s when some of the genes important in Drosophila development, most notably the various hox genes, were found to be broadly conserved across metazoans. Even some functions of these genes appeared to be conserved as vertebrate genes could sometimes substitute for missing fruit fly genes and vice versa. Such a high degree of genomic conservation was surprising given the obvious difference in morphological development between fruit flies and mice. How can conserved genes produce such morphological diversity? From this riddle the field of evolutionary developmental biology (evo-devo) was born.

Evo-devo has flourished in the last 15 years and now sports four specialized journals and considerable grant support. The future of evo-devo is currently the subject of debate, with authors discussing, for example, whether the promised synthesis of developmental genetics with evolutionary biology can actually be completed. With regard to neuroscience, however, the future of evo-devo is bright for evo-devo neurobiology is still young. Despite important early work on the evolution and development of eyes and the evolutionary inversion of the nervous system's dorsoventral position within the embryo (i.e. dorsal in vertebrates and ventral in arthropods), most of the variation in adult nervous systems has never been examined from an evo-devo perspective. Only in recent years have significant numbers of neuroscientists begun to ask how adult neural variation can be explained in terms of underlying developmental change.

To facilitate the further growth of evo-devo neurobiology, seven speakers gathered for a symposium entitled 'Developmental Mechanisms of Evolutionary Change in Nervous Systems' at the Euro-evo-devo meeting in Paris on July 9, 2010. These speakers were Markus Friedrich, Sophie Creuzet, Gerhard Schlosser, Yoshiyuki Yamamoto, Barbara Finlay, Todd Streelman, Loreta Medina, and myself. A subset of us wrote the review papers that are gathered in this special issue of Brain, Behavior and Evolution. The four sets of authors are: (1) Friedrich; (2) Medina, Bupesh, and Abellán; (3) Sylvester, Pottin, and Streelman, and (4) Charvet, Striedter, and Finlay. Instead of summarizing each paper individually, I discuss them in relation to some major themes or elements that can be traced through multiple papers.

One common theme in evo-devo work is that conserved similarities must be identified before taxon differences are revealed. Medina et al. provide an excellent example of how gene expression patterns can be used to identify homologous progenitor domains in diverse vertebrates. Their analysis suggests that the mammalian amygdala is generated from as many as seven different progenitor domains and that most of these can be ho-

\section{KARGER}

Fax +41613061234

E-Mail karger@karger.ch

www.karger.com
(C) 2011 S. Karger AG, Base

0006-8977/11/0783-0197\$38.00/0 
mologized across the major vertebrate taxa. By tracing the fate of cells generated from these precursor domains, Medina et al. have also shed new light on some controversial adult homologies. In addition, they have reconstructed some important phylogenetic changes in amygdala organization. They note, for example, that the amygdala of jawless vertebrates lacks an Nkx2-positive pallidal component and that the lateral pallial amygdala is significantly larger in mammals than in birds or amphibians.

A second element in many evo-devo studies is that developmental features are being compared across species even when those species develop at different rates. For example, many of the analyses discussed by Charvet et al. are based on comparisons between species with different gestation or incubation lengths. By taking such global changes in developmental speed into account, researchers can identify more specific changes in developmental timing or rates. Comparing ontogenies is more challenging when development is highly divergent between taxa, but it can nonetheless be done. For example, Friedrich's analysis of visual system reduction in fly larvae is based on comparing those larvae (i.e. maggots) to the pronymphal stages of grasshoppers and crickets, which look like miniature adults. Without this basis of comparison, the nervous system of Drosophila larvae would be difficult, if not impossible, to understand in evolutionary terms.

A third characteristic of much evo-devo research is that the line between quantitative and qualitative differences is fine. As Sylvester et al. review, eyelessness in adult cavefish is a qualitative feature (i.e. a loss of eyes) but the adult trait is based, at least in part, on quantitative changes in Hedgehog expression. Similarly, most of the gene expression changes discussed by Medina et al. are quantitative, but such quantitative changes probably produce adult amygdalae that are novel in the qualitative sense, containing subdivisions that they did not possess ancestrally. Although it is relatively easy to imagine how large quantitative changes can produce qualitative change (e.g. threshold effects), the two modes of change need not be tightly coupled. For example, Friedrich proposes that the miniature nervous system of fly larvae results from a massive quantitative reduction followed by a qualitative reorganization.

The fourth, nearly ubiquitous theme in evo-devo studies is the search for cellular and/or molecular causes of evolutionary change in developmental trajectories. For most neural characters, this quest for evo-devo mechanisms has barely begun. However, Sylvester et al. have identified some candidate genetic causes of evolutionary changes in brain patterning among a clade of African cichlids. Friedrich, too, has identified some genes that probably facilitated visual system reduction in fruit fly larvae. Most of the work discussed by Charvet et al. identifies evo-devo mechanisms at the cellular level as evolutionary changes in cell cycle rates and neurogenesis timing. Identifying the molecular causes of these cellular alterations is likely to be difficult given the plethora of genes involved in cell cycle control. However, explanations at the cellular level can be useful in and of themselves. One new question they raise is how developmental 'clocks' governing proliferation and differentiation can be independently controlled.

Beyond the search for specific evo-devo mechanisms, most evo-devo biologists also seek some general principles that govern evolutionary changes in genotype-tophenotype mappings. A common theme within this larger aim is the search for evo-devo 'constraints' that bias or limit the range of possible ontogenies. For example, Sylvester et al. note that the expansion of the Hedgehog domain in cavefishes reduces embryonic eye size but also leads to an expansion of basal forebrain tissues, including the olfactory bulb. Such pleiotropic effects are often assumed to be maladaptive, but enhanced olfaction is probably adaptive for cave-dwelling species. Charvet et al. also discuss the notion that evo-devo constraints need not be 'bad' and may themselves be shaped by natural selection, thereby leading to more 'evolvable' species. Moreover, constraints should not be viewed as totally rigid. For example, Charvet et al. explain that the developmental rule causing brain regions to vary allometrically with overall brain size can be combined with other modes of change.

Altogether, it is important to reiterate that evo-devo neurobiology is still in its juvenile stage. Many homologies have been resolved by comparing neural ontogenies, some developmental changes have been mechanistically explained, and a few principles are starting to emerge, but only the proverbial tip of the iceberg has been exposed. Hopefully the papers gathered here will stimulate more neuroscientists to join the evo-devo quest. That said, evodevo neurobiology cannot displace the functional analysis of how evolutionary changes in nervous system organization affect animal behavior and ecology. Evo-devo and functional analyses are different sides of the same coin which must be synthesized. That, too, remains an exciting challenge.

Georg F. Striedter 